\title{
POSITIVE PERIODIC SOLUTIONS OF SECOND-ORDER DIFFERENTIAL EQUATIONS WITH SINGULARITIES
}

\author{
HONG-XU LI
}

(Received 17 October 2007)

\begin{abstract}
In this work, we consider the periodic boundary value problem

$$
\left\{\begin{array}{l}
x^{\prime \prime}+a(t) x=f(t, x)+c(t) \\
x(0)=x(T), \quad x^{\prime}(0)=x^{\prime}(T)
\end{array}\right.
$$

where $a, c \in L^{1}(0, T)$ and $f$ is a Carathéodory function. An existence theorem for positive periodic solutions is proved in the case where the associated Green function is nonnegative. Our result is valid for systems with strong singularities, and answers partially the open problem raised in Torres ['Weak singularities may help periodic solutions to exist', J. Differential Equations 232 (2007), 277-284].
\end{abstract}

2000 Mathematics subject classification: 34B15, 34B16.

Keywords and phrases: positive periodic solution, Green's function, singularity.

\section{Introduction and preliminaries}

Consider the periodic boundary value problem

$$
\begin{gathered}
x^{\prime \prime}+a(t) x=f(t, x)+c(t), \\
x(0)=x(T), \quad x^{\prime}(0)=x^{\prime}(T),
\end{gathered}
$$

where $a, c \in L^{1}(0, T)$ and $f:[0, T] \times(0, \infty) \rightarrow \mathbb{R}$ is a function of $L^{1}$-Carathéodory type, that is, it is continuous in the second variable and for every $0<r<s$ there exists $h_{r, s} \in L^{1}(0, T)$ such that $|f(t, x)| \leq h_{r, s}(t)$ for all $x \in[r, s]$ and almost every $t \in$ $[0, T]$. We are interested in equation (1) with a singularity in $x=0$, which means

$$
\lim _{x \rightarrow 0+} f(t, x)=+\infty \quad \text { uniformly almost every } t \in[0, T] .
$$

The singularities appear when electrostatic or gravitational forces are considered.

In recent years, the periodic boundary value problem (1)-(2) has attracted attention from many specialists in differential equations. Lazer and Solimini [6] studied this

(c) 2008 Australian Mathematical Society 0004-9727/08 \$A2.00+0.00 
problem in the special case $f(t, x)=1 / x^{\lambda}$. They showed that the strong force assumption $\lambda \geq 1$ is necessary in some sense for the existence of positive periodic solutions. Since then, the strong force condition has become standard in related work; see, for instance, $[1,3-5,7-9,13,14]$ and the references therein. Compared with the case of strong singularities, the study of the existence of periodic solutions under the presence of weak singularities is much more recent. Here we refer the reader to $[2,9-11]$.

In [12], the boundary value problem (1)-(2) is studied by using Schauder's fixed point theorem, and some existence results prove that in some situations weak singularities may help create periodic solutions. However, 'the validity of such results under a strong force assumption remains as an open problem' (see [12]). The purpose of this paper is to give a partial answer to this open problem, namely, we prove that [12, Theorem 2] is valid under a strong force assumption if $a \succ 0$ and $\|a\|_{1} T<1$ (see Theorem 1 below).

Let us fix some notation. Given $a \in L^{1}(0, T)$, we write $a \succ 0$ if $a \geq 0$ for almost every $t \in[0, T]$ and it is positive in a set of positive measure. We denote by $a_{*}$ and $a^{*}$ the essential infimum and supremum of $a$, respectively. The usual supremum norm and $L^{1}$-norm are denoted by $\|\cdot\|$ and $\|\cdot\|_{1}$, respectively.

\section{Main results}

Throughout this paper, we assume the following hypothesis:

(H1) Green's function $G(t, s)$ of the linear equation $x^{\prime \prime}+a(t) x=0$ with periodic condition (2) is nonnegative for every $(t, s) \in[0, T] \times[0, T]$.

Under condition (H1), the unique $T$-periodic solution of $x^{\prime \prime}(t)+a(t) x=h(t)$ is given by

$$
x(t)=\int_{0}^{T} G(t, s) h(s) d s
$$

We remark that, when $a(t)=k^{2}>0$, it is not hard to see that condition (H1) is equivalent to $0<k^{2} \leq(\pi / T)^{2}$. For a nonconstant $a(t)$, we refer to an $L^{p}$-criterion given as [12, Lemma 1], where the condition $a \succ 0$ is needed. So the assumption $a \succ 0$ in this paper is natural.

The following theorem is [12, Theorem 1], and will be used in the proof of our main result.

THEOREM A. Assume (H1) and that there exist $b \succ 0$ and $\lambda>0$ such that

$$
0 \leq f(t, x) \leq \frac{b(t)}{x^{\lambda}} \quad \text { for all } x>0, \text { for almost every } t .
$$

If $\gamma_{*}>0$, then there exists a positive $T$-periodic solution of (1). 
Lemma 1. Assume that $a \succ 0$. There exists $0<\eta<1$ such that, for any $t_{0}, t_{1} \in$ $[0, T]$,

$$
0 \leq \int_{t_{0}}^{t_{1}} \int_{t_{0}}^{r} a(s) d s d r \leq \eta T\|a\|_{1} .
$$

PROOF. The first part of inequality (3) is clear. So it suffices to prove the second part. We can easily see that there exist $T_{1}, T_{2}, T_{3} \in(0, T), T_{1}<T_{2}<T_{3}$, such that

$$
\int_{0}^{T_{i}} a(s) d s=\frac{i\|a\|_{1}}{4}, \quad i=1,2,3 .
$$

Let $l=\min \left\{T_{1}, T_{2}-T_{1}, T_{3}-T_{2}, T-T_{3}\right\}$. For any $t \in[0, T]$, set

$$
A(t)=\left\{\delta(t):\left|\int_{t}^{t+\delta(t)} a(s) d s\right|=\frac{\|a\|_{1}}{2}\right\} .
$$

Then $|\delta(t)| \geq l$ for any $\delta(t) \in A(t), t \in[0, T]$.

Without loss of generality, we may assume that $t_{0}<t_{1}$. If there does not exist any $\delta\left(t_{0}\right)$ in $A\left(t_{0}\right)$ such that $t_{0}+\delta\left(t_{0}\right) \in\left[t_{0}, t_{1}\right]$, we can get easily that, for any $r \in\left[t_{0}, t_{1}\right]$, $\int_{t_{0}}^{r} a(s) d s \leq\|a\|_{1} / 2$. So

$$
\int_{t_{0}}^{t_{1}} \int_{t_{0}}^{r} a(s) d s d r \leq \frac{1}{2} T\|a\|_{1} .
$$

If there exists $\delta\left(t_{0}\right) \in A\left(t_{0}\right)$ such that $t_{0}+\delta\left(t_{0}\right) \in\left[t_{0}, t_{1}\right]$, then $\delta\left(t_{0}\right)>l$, and

$$
\begin{aligned}
\int_{t_{0}}^{t_{1}} \int_{t_{0}}^{r} a(s) d s d r & =\left(\int_{t_{0}}^{t_{0}+\delta\left(t_{0}\right)}+\int_{t_{0}+\delta\left(t_{0}\right)}^{t_{1}}\right) \int_{t_{0}}^{r} a(s) d s d r \\
& \leq \delta\left(t_{0}\right)\|a\|_{1} / 2+\left|t_{1}-t_{0}-\delta\left(t_{0}\right)\right|\|a\|_{1} \\
& \leq \delta\left(t_{0}\right)\|a\|_{1} / 2+\left(T-\delta\left(t_{0}\right)\right)\|a\|_{1} \\
& =\left(T-\delta\left(t_{0}\right) / 2\right)\|a\|_{1} \\
& \leq(T-l / 2)\|a\|_{1} .
\end{aligned}
$$

Let $\eta=\max \{(1 / 2), 1-(l / 2 T)\}$. Then (3) is true.

Now we are in a position to give our main result.

THEOREM 1. Let us assume (H1) and that there exist $b, \hat{b} \succ 0$ and $\lambda>0$ such that

$$
0 \leq \frac{\hat{b}(t)}{x^{\lambda}} \leq f(t, x) \leq \frac{b(t)}{x^{\lambda}} \text { for all } x>0, \text { for almost every } t .
$$

If $\gamma_{*}=0, a \succ 0$ and $T\|a\|_{1}<1$, then there exists a positive $T$-periodic solution of (1).

PROOF. We consider the following family of systems,

$$
x^{\prime \prime}+a(t) x(t)=f(t, x)+c(t)+\frac{a(t)}{n},
$$


with $n$ any natural number. Noticing that $\int_{0}^{T} G(t, s) a(s) d s=1$ and replacing $c(t)$ by $c(t)+a(t) / n$, it is easy to get from Theorem A that there exists a positive $T$-periodic solution $x_{n}$ of (4) for each $n$.

For the sake of convenience, we assume that

$$
\begin{gathered}
\gamma(t)=\int_{0}^{T} G(t, s) c(s) d s, \quad \beta(t)=\int_{0}^{T} G(t, s) b(s) d s \\
\hat{\beta}(t)=\int_{0}^{T} G(t, s) \hat{b}(s) d s .
\end{gathered}
$$

Now we complete the proof in the following two steps.

STEP 1. Suppose that $x_{n}(t) \geq\left(1-\|a\|_{1} T\right)\left\|x_{n}\right\|$ for all $t \in[0, T]$. Clearly, the $T$ periodic solution $x_{n}$ of (4) satisfies

$$
x_{n}(t)=\int_{0}^{T} G(t, s)\left[f\left(s, x_{n}(s)\right)+c(s)+a(s) / n\right] d s .
$$

So by (H2),

$$
\begin{aligned}
x_{n}(t) & \leq \int_{0}^{T} G(t, s) f\left(s, x_{n}(s)\right) d s+\gamma(t)+1 / n \\
& \leq \int_{0}^{T} \frac{G(t, s) b(s)}{x_{n}^{\lambda}(s)} d s+\gamma(t)+1 / n \\
& \leq \frac{\beta^{*}}{\left(\left(1-\|a\|_{1} T\right)\left\|x_{n}\right\|\right)^{\lambda}}+\gamma^{*}+1 .
\end{aligned}
$$

This implies that the sequence $\left\{x_{n}\right\}$ is uniformly bounded, that is, there exists $R<\infty$ such that $\left\|x_{n}\right\| \leq R$ for all $n$. As a result,

$$
\begin{aligned}
x_{n}(t) & =\int_{0}^{T} G(t, s)\left[f\left(s, x_{n}(s)\right)+c(s)+a(s) / n\right] d s \\
& \geq \int_{0}^{T} G(t, s) \frac{\hat{b}(s)}{R^{\lambda}} d s \\
& \geq \frac{\hat{\beta}_{*}}{R^{\lambda}}=: r .
\end{aligned}
$$

We note that $\hat{\beta}_{*}>0$ as a consequence of (H1) and $\hat{b} \succ 0$. Thus $0<r \leq x_{n}(t) \leq R$ for all $n$ and $t \in[0, T]$.

On the other hand, since $x_{n}^{\prime}(0)=x_{n}^{\prime}(T)$, by (4),

$$
\int_{0}^{T} a(s) x_{n}(s) d s=\int_{0}^{T}\left[f\left(s, x_{n}(s)\right)+c(s)+a(s) / n\right] d s .
$$


Meanwhile, there exists $t_{n 0} \in[0, T]$ such that $x_{n}^{\prime}\left(t_{n 0}\right)=0$ since $x_{n}(0)=x_{n}(T)$. Then, by (4) again,

$$
\left.x_{n}^{\prime}(t)=\int_{t_{n 0}}^{t}\left[f\left(s, x_{n}(s)\right)+c(s)+a(s) / n-a(s) x_{n}(s)\right)\right] d s .
$$

Hence,

$$
\begin{aligned}
\left\|x_{n}^{\prime}\right\| & =\max _{t \in[0, T]}\left|\int_{t_{n 0}}^{t}\left[f\left(s, x_{n}(s)\right)+c(s)+a(s) / n-a(s) x_{n}(s)\right] d s\right| \\
& \leq \int_{0}^{T}\left[f\left(s, x_{n}(s)\right)+c(s)+a(s) / n\right] d s+\int_{0}^{T} a(s) x_{n}(s) d s \\
& =2 \int_{0}^{T} a(s) x_{n}(s) d s \\
& \leq 2 R\|a\|_{1} .
\end{aligned}
$$

Therefore, the sequence $\left\{x_{n}\right\}$ is uniformly bounded and equicontinuous. By the Arzelà-Ascoli theorem, there exists a subsequence $\left\{x_{n_{k}}\right\}$ of $\left\{x_{n}\right\}$ such that $\left\{x_{n_{k}}\right\}$ converges uniformly to a continuous function $x$, and $0<r \leq x(t) \leq R, t \in[0, T]$. Moreover,

$$
\begin{aligned}
x_{n_{k}}(t) & =\int_{0}^{T} G(t, s)\left[f\left(s, x_{n_{k}}(s)\right)+c(s)+a(s) / n\right] d s \\
& \rightarrow \int_{0}^{T} G(t, s)[f(s, x(s))+c(s)] d s
\end{aligned}
$$

as $k \rightarrow \infty$. So $x(t)$ is a positive $T$-periodic solution of (1).

STEP 2. Suppose that $x_{n}(t)<\left(1-\|a\|_{1} T\right)\left\|x_{n}\right\|$ for some $t \in[0, T]$. We first prove that there exists $t_{n 0} \in[0, T]$ such that

$$
x_{n}\left(t_{n 0}\right)=\left\|x_{n}\right\|, \quad x_{n}^{\prime}\left(t_{n 0}\right)=0 .
$$

If $x_{n}(0) \neq\left\|x_{n}\right\|$, it is clear that there exists $t_{n 0} \in(0, T)$ such that (5) holds. If $x_{n}(0)=\left\|x_{n}\right\|$, it is sufficient to prove that $x_{n}^{\prime}(0)=0$. In fact, we can see easily that $x_{n}^{\prime}(0)>0$ contradicts the fact that 0 is the maximum point of $x_{n}$. If $x_{n}^{\prime}(0)<0$, then $x_{n}^{\prime}(T)=x_{n}^{\prime}(0)<0$, which is also in contradiction to $x_{n}(0)=x_{n}(T)=\left\|x_{n}\right\|=$ $\max \left\{\left|x_{n}(t)\right|: t \in[0, T]\right\}$. Therefore, $x_{n}^{\prime}(0)=0$, and (5) is true.

Meanwhile, it follows from the assumption of this step that there exists $t_{n 1} \in[0, T]$ such that $x_{n}\left(t_{n 1}\right)=\left(1-\|a\|_{1} T\right)\left\|x_{n}\right\|$ and $x_{n}(t) \geq\left(1-\|a\|_{1} T\right)\left\|x_{n}\right\|$ if $t$ is between $t_{n 0}$ and $t_{n 1}$. Then by (4) and (5),

$$
x_{n}^{\prime}(t)=\int_{t_{n 0}}^{t}\left[f\left(s, x_{n}(s)\right)+c(s)+a(s) / n-a(s) x_{n}(s)\right] d s
$$


and

$$
x_{n}(t)-\left\|x_{n}\right\|=\int_{t_{n 0}}^{t} \int_{t_{n 0}}^{r}\left[f\left(s, x_{n}(s)\right)+c(s)+a(s) / n-a(s) x_{n}(s)\right] d s d r .
$$

Therefore, by Lemma 1 we can get

$$
\begin{aligned}
\|a\|_{1} T\left\|x_{n}\right\| & =\left|x_{n}\left(t_{n 1}\right)-\left\|x_{n}\right\|\right| \\
& =\left|\int_{t_{n 0}}^{t_{n 1}} \int_{t_{n 0}}^{r}\left[f\left(s, x_{n}(s)\right)+c(s)+a(s) / n-a(s) x_{n}(s)\right] d s d r\right| \\
& \leq \int_{t_{n 0}}^{t_{n 1}} \int_{t_{n 0}}^{r}\left[\frac{b(s)}{x_{n}^{\lambda}(s)}+c(s)+\frac{a(s)}{n}\right] d s d r+\int_{t_{n 0}}^{t_{n 1}} \int_{t_{n 0}}^{r} a(s) x_{n}(s) d s d r \\
& \leq \int_{t_{n 0}}^{t_{n 1}}\left(\frac{\|b\|_{1}}{\left(\left(1-\|a\|_{1} T\right)\left\|x_{n}\right\|\right)^{\lambda}}+\|c\|_{1}+\|a\|_{1}\right) d r+\eta T\|a\|_{1}\left\|x_{n}\right\| \\
& \leq\left(\frac{\|b\|_{1}}{\left(\left(1-\|a\|_{1} T\right)\left\|x_{n}\right\|\right)^{\lambda}}+\|c\|_{1}+\|a\|_{1}\right) T+\eta T\left\|_{1}\right\|_{1}\left\|x_{n}\right\|,
\end{aligned}
$$

that is,

$$
(1-\eta)\|a\|_{1}\left\|x_{n}\right\| \leq \frac{\|b\|_{1}}{\left(\left(1-\|a\|_{1} T\right)\left\|x_{n}\right\|\right)^{\lambda}}+\|c\|_{1}+\|a\|_{1} .
$$

This also implies the uniform boundedness of $\left\{x_{n}\right\}$ since $\lambda>0$, and by the same argument at the end of step 1 we can get a positive $T$-periodic solution of (1). The proof is complete.

REMARK. Theorem 1 gives a partial answer to the open problem given in [12]: our result is valid under the strong force assumption $\lambda \geq 1$ if $a \succ 0$ and $\|a\|_{1} T<1$. However, the case $\|a\|_{1} T \geq 1$ under the strong force assumption still remains an open problem.

\section{References}

[1] D. Bonheure, C. Fabry and D. Smets, 'Periodic solutions of forced isochronous oscillators at resonance', Discrete Contin. Dyn. Syst. 8 (2002), 907-930.

[2] J. Chu, P. J. Torres and M. Zhang, 'Periodic solutions of second order non-autonomous singular dynamical systems', J. Differential Equations 239(1) (2007), 196-212.

[3] A. Fonda, R. Manásevich and F. Zanolin, 'Subharmonic solutions for some second order differential equations with singularities', SIAM J. Math. Anal. 24 (1993), 1294-1311.

[4] P. Habets and L. Sanchez, 'Periodic solutions of some Linard equations with singularities', Proc. Amer. Math. Soc. 109 (1990), 1135-1144.

[5] D. Jiang, J. Chu and M. Zhang, 'Multiplicity of positive periodic solutions to superlinear repulsive singular equations', J. Differential Equations 211(2) (2005), 282-302.

[6] A. C. Lazer and S. Solimini, 'On periodic solutions of nonlinear differential equations with singularities', Proc. Amer. Math. Soc. 99 (1987), 109-114.

[7] M. del Pino and R. Manásevich, 'Infinitely many T-periodic solutions for a problem arising in nonlinear elasticity', J. Differential Equations 103 (1993), 260-277. 
[8] I. Rachunková, S. Stanek and M. Tvrdý, Singularities and Laplacians in Boundary Value Problems for Nonlinear Ordinary Differential Equations, Handbook of Differential Equations (Ordinary Differential Equations), 3 (Elsevier, Amsterdam, 2006).

[9] I. Rachunková, M. Tvrdý and I. Vrkoč, 'Existence of nonnegative and nonpositive solutions for second order periodic boundary value problems', J. Differential Equations 176 (2001), 445-469.

[10] M. Ramos and S. Terracini, 'Noncollision periodic solutions to some singular dynamical systems with very weak forces', J. Differential Equations 118 (1995), 121-152.

[11] P. J. Torres, 'Existence of one-signed periodic solutions of some second order differential equations via a Krasnoselskii fixed point theorem', J. Differential Equations 190 (2003), 643-662.

[12] - 'Weak singularities may help periodic solutions to exist', J. Differential Equations 232 (2007), 277-284.

[13] P. J. Torres and M. Zhang, 'Twist periodic solutions of repulsive singular equations', Nonlinear Anal. 56 (2004), 591-599.

[14] M. Zhang, 'A relationship between the periodic and the Dirichlet BVPs of singular differential equations', Proc. Roy. Soc. Edinburgh Sect. A 128 (1998), 1099-1114.

HONG-XU LI, Department of Mathematics, Sichuan University, Chengdu, Sichuan 610064, PR China

e-mail: hoxuli@sohu.com 